\title{
Actitudes y desafíos percibidos de médicos residentes de medicina familiar hacia la investigación: un estudio piloto de Paraguay, 2016
}

\author{
Julio Torales ${ }^{\mathrm{I}}$, Iván Barrios ${ }^{\mathrm{II}}$, Cinthia Camacho ${ }^{\mathrm{III}}$, Andrés Szwako ${ }^{\mathrm{III}}$, Angélica Samudio ${ }^{\mathrm{I}}$, Margarita \\ Samudio ${ }^{\text {IV }}$, Sergio Aquinov \\ IDirección de Investigaciones, Facultad de Ciencias Médicas, Universidad Nacional de Asunción (Paraguay). \\ II Facultad de Ciencias Médicas, Universidad Nacional de Asunción (Paraguay). \\ III Cátedra de Medicina Familiar, Facultad de Ciencias Médicas, Universidad Nacional de Asunción (Paraguay). \\ IV Instituto de Investigaciones en Ciencias de la Salud, Universidad Nacional de Asunción (Paraguay). \\ V Cátedra de Neumología, Facultad de Ciencias Médicas. Universidad Nacional de Asunción (Paraguay)
}

Cómo referenciar este artículo/

How to reference this article:
Torales J, Barrios I, Camacho C, Szwako A, Samudio A, Samudio M, Aquino S. Actitudes y desafíos percibidos de médicos residentes de medicina familiar hacia la investigación: un estudio piloto de Paraguay, 2016Mem. Inst. Investig. Cienc. Salud. 2016;14(3): 107-114

\section{R E S U M E N}

Las competencias en investigación han sido reconocidas como parte esencial de la educación médica en el postgrado. El objetivo de este estudio piloto fue evaluar las actitudes y desafíos percibidos de médicos residentes de Medicina Familiar hacia la investigación. Estudio piloto, observacional, descriptivo, de corte transverso y muestreo no probabilístico, en el que se incluyeron 20 médicos residentes. Se utilizó un cuestionario sobre actitudes y desafíos percibidos sobre la investigación. Se realizó estadística descriptiva para todas las variables. Los resultados se expresaron en frecuencias, porcentajes, medias y desvíos estándar. Del total de participantes, sólo 7 (35\%) participaron en algún proyecto de investigación durante la residencia, principalmente, en estudios descriptivos (30\%) y reportes de casos (15\%). El $60 \%$ respondió que en la residencia no desarrollaban un programa específico de formación en investigación, aunque la mayoría estuvo de acuerdo con que la investigación es esencial para el desarrollo de una carrera académica $(95 \%)$, que mejora la atención sanitaria del paciente $(75 \%)$ y que su estudio debe ser obligatorio en el currículo académico (70\%). Los médicos residentes paraguayos presentan actitudes positivas y reconocen la importancia de la investigación, no obstante, sólo el $35 \%$ ha realizado investigaciones durante su programa de residencia, siendo el principal desafío al que se enfrentan, la falta detiempo protegido. Se considera necesario el rediseño del currículo del programa de residencia en Medicina Familiar de la Universidad Nacional de Asunción a fin de incluir de manera trasversal la formación en investigación de médicos residentes.

Palabras clave: investigación, evaluación, actitudes, desafíos, médicos residentes, medicina familiar.

\section{Attitudes and perceived challenges towards research of medical residents of family medicine: a pilot study from Paraguay, 2016}

\section{A B S T R A C T}

Research skills have been recognized as an essential part of medical education at the postgraduate level. The objective of this pilot study was to evaluate the attitudes and perceived challenges of family medicine residents toward research. This was an observational, descriptive and cross-sectional pilot study, of non-probabilistic sampling, 
which included 20 medical residents. A questionnaire on attitudes and perceived challenges on research was used. Descriptive statistics were performed for all variables. The results were expressed as frequencies, percentages, means and standard deviations. Of the total participants, only $7(35 \%)$ participated in a research project during residency, mainly in descriptive studies (30\%) and case reports (15\%). Sixty percent of participants responded that their residency did not develop a specific program of research training, although most agreed that research was essential for the development of an academic career (95\%), improved patient healthcare $(75 \%)$ and that the study of research techniques should be mandatory in the academic curriculum (70\%). Paraguayan medical residents have positive attitudes and recognize the importance of research. However, only $35 \%$ have conducted research during their residency program; being the main challenge they face, the need for a protected time for research. It is considered necessary to redesign the curriculum of the Family Medicine residency program of the National University of Asuncion, in order to include research training in a transversal way.

Keywords: research, evaluation, attitudes, challenges, medical residents, family medicine.

\section{INTRODUCCIÓN}

La educación en habilidades de investigación es reconocida como parte fundamental de un programa de residencia médica y, además, las actividades de investigación representan un importante aspecto del entrenamiento que todo médico residente debe recibir, puesto que mejoran el aprendizaje, el pensamiento crítico, la atención de los pacientes y la satisfacción con el programa de residencia(1-7).

Las competencias en investigación han sido reconocidas como parte esencial de la educación médica en el postgrado (8-11). Investigar durante los programas de residencia médica se constituye en un desafío por diversas razones relacionadas con las actitudes de los propios médicos residentes, sus unidades formadoras y su propio plan de estudios $(12,13)$.

En la Universidad Nacional de Asunción, Paraguay, las actividades de investigación todavía no están lo suficientemente incorporadas en los planes de estudio de las residencias médicas, como por ejemplo, la Residencia Médica de Medicina Familiar. En esta última, si bien la presentación de una investigación es condición requerida para optar al título de Especialista en Medicina Familiar, el entrenamiento en investigación no está suficientemente incorporado al plan de estudios (12). Una revisión de la literatura muestra que no se conoce aún nada acerca del conocimiento y actitudes sobre la investigación entre médicos residentes de Medicina Familiar de la Universidad Nacional de Asunción, así como los desafíos a los que se enfrentan.

En este sentido, el objetivo de este estudio piloto fue evaluar las actitudes y desafíos percibidos de médicos residentes de Medicina Familiar hacia la investigación. Se espera que los resultados de este trabajo ayuden a identificar las dificultades y desafíos enfrentados por los médicos para realizar investigaciones durante la residencia, así como a contribuir en la construcción y facilitación de planes de estudio que incluyan a la investigación como elemento clave de las residencias médicas.

\section{MATERIALES Y MÉTODOS \\ Diseño}

El presente fue un estudio piloto, observacional, descriptivo y de corte transverso.

\section{Población en estudio y recolección de datos}

Los participantes del estudio fueron médicos residentes de Medicina Familiar, que cumplían funciones en el Hospital de Clínicas o en el Sanatorio Adventista de Asunción, y que se inscribieron voluntariamente para participar del estudio. Se debe recalcar que ambos programas de residencia se encuentran avalados por la Universidad Nacional de Asunción. Un cuestionario fue completado por todos los participantes del estudio, bajo la supervisión del investigador principal (autor correspondiente) del presente estudio. El tiempo otorgado para llenar el cuestionario estuvo limitado a 15 minutos. Todos los participantes tenían al español como lengua materna, idioma en el que estaba escrito el cuestionario.

\section{Cuestionario}

El cuestionario (Tabla 1) incluyó una sección con 9 preguntas relacionadas a los detalles personales de los participantes, su año de graduación, su año de residencia, el Hospital en 
dónde prestaban servicios y sus antecedentes de participación en proyectos de investigación realizados durante su residencia médica; en una segunda parte, el cuestionario incluyó una sección acerca de actitudes y desafíos percibidos por los participantes acerca de la investigación.

Tabla 1. Cuestionario.

1. Sexo: Masculino/Femenino

2. Edad: _años

3. Año de graduación de la carrera de Medicina:

4. Universidad de la cual se graduó:

5. Año actual de Residencia:

6. Lugar donde realiza su Residencia:

7. Universidad que avala su postgrado:

8. ¿Ha participado (o está participando) de algún proyecto de investigación durante su residencia? Sí---¿Cuántas veces?---No

9. En caso que haya respondido SI en la pregunta 8, por favor responda las preguntas 9a, 9b, 9c sobre las características del proyecto de investigación:

9.a. Tipo de proyecto (puede marcar más de una opción y coloque al lado cuantas veces, en caso necesario): Estudio descriptivo (por ejemplo de prevalencia, de morbilidad, encuestas) ---Estudio de cohortes---Estudio de casos y controles---Ensayo clínico---Revisión de la literatura--Reporte de caso.

9.b. ¿Cuál fue su participación en el proyecto de investigación? (puede macar más de una opción): Contribuí con el concepto (diseño) del proyecto--Contribuí con revisión de la literatura---Contribuí con la recolección de datos--Contribuí con el análisis de datos---Contribuí con el manuscrito final.

9.c. ¿Se publicó los resultados de la investigación en alguna Revista Científica? Sí---Nombrar la revista---No

10.Marqué la opción que se ajuste al programa de Residencia que usted está cursando:

- ¿Tiene su programa de residencia un módulo específico sobre metodología de la investigación? Sí/No

- ¿Es obligatorio presentar al menos una investigación para concluir su programa de residencia? Sí/No

- ¿Le asigna su programa de residencia un tutor/orientador para guiarlo en su/s proyecto/s de investigación? Sí/No

11.De la siguiente tabla, marque lo que opina sobre algunas aseveraciones:

-El entrenamiento en investigación debe ser obligatorio en el programa de residencia de Medicina Familiar: De acuerdo---Neutral---En desacuerdo

-La investigación mejora la atención sanitaria del paciente: De acuerdo--Neutral---En desacuerdo

-La investigación es esencial para el desarrollo de una carrera docente o académica: De acuerdo---Neutral---En desacuerdo

-El entrenamiento en investigación compite con el tiempo del residente, por lo que interfiere con las competencias médicas a ser adquiridas: De acuerdo--Neutral---En desacuerdo

12.En la siguiente tabla, valore de 1 al 5 (siendo 1 lo menos importante y 5 lo más importante), los siguientes desafíos que deben ser superados para incentivar la investigación en la residencia:

-Falta de tiempo protegido para la investigación

-Falta de conocimientos y habilidades en investigación

-Falta de tutores

-Falta de interés

-Falta de ideas de investigación

-Falta de apoyo institucional/recursos económicos 
A fin de asegurar la validez del cuestionario, las preguntas relacionadas con epidemiología y estadística se compartieron con los miembros de la Dirección de Investigaciones de la Facultad de Ciencias Médicas de la Universidad Nacional de Asunción. Todos ellos coincidieron en que las preguntas estaban bien formuladas.

\section{Análisis estadístico}

Los datos fueron analizados utilizando el software EpiInfo 7.2 versión libre (Centers for Disease Control and Prevention, Atlanta, Estados Unidos de América). Se realizó estadística descriptiva para todas las variables. Los resultados se expresaron en frecuencias, porcentajes, medias y desvíos estándar.

\section{Tamaño de la muestra}

En estudio piloto se incluyeron 20 médicos residentes de Medicina Familiar.

\section{Aspectos éticos}

El presente trabajo fue aprobado por el Departamento de Normas de la Dirección de Investigaciones de la Facultad de Ciencias Médicas de la Universidad Nacional de Asunción. Los participantes firmaron un consentimiento libre, voluntario, informado y escrito. Los cuestionarios se llenaron de forma anónima.

\section{RESULTADOS}

El $55 \%$ de los participantes fue del sexo femenino, con edades comprendidas entre los 27 y 40 años; observamos una media de $31 \pm 3$ años. El $55 \%$ se gradúo como médico de la Universidad Nacional de Asunción y el $50 \%$ se encontraba realizando su primer año de residencia, el $65 \%$ de ellos en el Hospital de Clínicas de la Universidad Nacional de Asunción. El resto de los datos socio-académicos puede observarse en la Tabla 2.

Tabla 2. Perfil socio-académico

\begin{tabular}{lll}
\hline Variable & $\mathbf{n}$ & \% \\
\hline Sexo & 9 & 55 \\
Femenino & 11 & 45 \\
Masculino & & \\
Año de graduación & 1 & 5 \\
2008 & 2 & 10 \\
2009 & 1 & 5 \\
2011 & 4 & 20 \\
2012 & 7 & 35 \\
2013 & 5 & 25 \\
2014 & & \\
Universidad de donde se graduó & & \\
Universidad del Pacífico & 1 & 1 \\
Universidad Católica de Villarrica & 2 & 2 \\
Universidad Nacional de Asunción & 11 & 11 \\
Universidad Nacional de Concepción & 1 & 1 \\
Universidad Nacional del Este & 2 & 2 \\
Universidad del Norte & 3 & 3 \\
Año de residencia médica & & \\
Primero & 10 & 50 \\
Segundo & 3 & 15 \\
Tercero & 7 & 35 \\
\hline & & \\
\hline & &
\end{tabular}


De los 20 residentes que participaron, 7 (35\%) habían participado en algún proyecto de investigación durante la residencia, mientras que $13(65 \%)$ en ninguno. Médicos residentes de segundo y tercer año fueron los únicos que habían realizado algún tipo de trabajo.

De los 7 residentes que habían participado en investigaciones durante la residencia, el 5\% se involucró en el diseño del proyecto, un $25 \%$ en la revisión de literatura, un $30 \%$ en la recolección de los datos, un $25 \%$ en el análisis de los datos y un $10 \%$ participó de la redacción del manuscrito final. Los tipos de investigación en los cuales los médicos residentes habían participado incluían principalmente estudios descriptivos (30\%) y reportes de casos (15\%). Al consultar sobre si en la residencia desarrollaban un programa específico de formación en metodología de la investigación, el $60 \%$ respondió que no, aunque el $95 \%$ afirmó que debía presentarse al menos un trabajo de investigación para concluir el programa de residencia.

La mayoría de los médicos residentes estaba de acuerdo con que la investigación es esencial para el desarrollo de una carrera académica (95\%), que la misma mejora la atención sanitaria del paciente $(75 \%)$ y que el estudio sobre metodología de la investigación debe ser obligatorio en el programa académico de la residencia en Medicina Familiar (70\%). En la Tabla 3 puede observar la actitud hacia la investigación por parte de los residentes de Medicina Familiar.

Tabla 3. Actitudes

\begin{tabular}{llll}
\hline Afirmación & $\begin{array}{l}\text { De acuerdo } \\
\mathbf{n}(\%)\end{array}$ & $\begin{array}{l}\text { Neutral } \\
\mathbf{n}(\%)\end{array}$ & $\begin{array}{l}\text { En } \\
\text { desacuerdo } \\
\mathbf{n}(\%)\end{array}$ \\
\hline $\begin{array}{l}\text { El entrenamiento en investigación deber ser } \\
\text { obligatorio en el programa de residencia de } \\
\text { medicina familiar }\end{array}$ & $14(70)$ & $5(25)$ & $1(5)$ \\
$\begin{array}{l}\text { La investigación mejora la atención sanitaria } \\
\text { del paciente }\end{array}$ & $15(75)$ & $3(15)$ & $2(10)$ \\
$\begin{array}{l}\text { La investigación es esencial para el desarrollo } \\
\text { de una carrera docente o académica }\end{array}$ & $19(95)$ & $1(5)$ & $0(0)$ \\
$\begin{array}{l}\text { El entrenamiento en investigación compite con } \\
\text { el tiempo del residente, por lo que interfiere } \\
\text { con las competencias médicas a ser adquiridas }\end{array}$ & $9(45)$ & $6(30)$ & $4(20)$ \\
\hline
\end{tabular}

En cuanto a los desafíos que los residentes encuentran en su formación, se utilizó un sistema de calificación donde el 5 significó más importante y el 1 menos importante, obteniéndose las medias expuestas en la tabla 4. En la misma se observa que el mayor desafío para los médicos residentes de Medicina Familiar paraguayos que participaron de este estudio era contar con un tiempo destinado a la investigación y, en segundo lugar, la falta de conocimientos y habilidades en investigación.

Tabla 4. Desafíos

\begin{tabular}{lll}
\hline Desafío & Media & $\begin{array}{l}\text { Desviación } \\
\text { estándar }\end{array}$ \\
\hline Falta tiempo protegido para la investigación & 4,45 & 0,759 \\
Falta de conocimientos y habilidades en investigación & 4,20 & 0,951 \\
Falta de tutores & 2,85 & 1,182 \\
Falta de interés & 2,90 & 1,210 \\
Falta de ideas de investigación & 3,45 & 1,191 \\
Falta de apoyo institucional/recursos económicos & 3,60 & 1,314 \\
\hline
\end{tabular}




\section{DISCUSIÓN}

Nuestro estudio se enfocó en las actitudes y desafíos percibidos sobre la investigación en médicos residentes de Medicina Familiar, de Asunción - Paraguay. La investigación durante la residencia no sólo puede promover el interés por una carrera académica o docente específica, sino también puede hacer que los residentes sean más competitivos para obtener becas y cargos académicos $(14,15)$. El entrenamiento en investigación en Paraguay está actualmente siendo incorporado, paulatinamente, como parte del currículo de las escuelas de medicina y los programas de residencia, a fin de contribuir a la generación de médicos científicos competentes (16). La educación médica intenta preparar a los médicos para que los mismos hagan frente a los desafíos de la práctica clínica, a través del reforzamiento de sus roles de clínicos, educadores e investigadores (17).

Los datos publicados sobre las actitudes de médicos residentes hacia la investigación, en diferentes especialidades, sugieren que no todos los residentes están entusiasmados con la investigación y que algunos de ellos no la valoran como una experiencia académica útil dentro de la residencia médica (18-25). Esto está en contraste con nuestros resultados, en donde la mayoría de los residentes estaba de acuerdo con que la investigación es esencial para el desarrollo de una carrera académica (95\%), que la misma mejora la atención sanitaria del paciente $(75 \%)$ y que el estudio sobre metodología de la investigación debe ser obligatorio en el programa académico de la residencia en Medicina Familiar (70\%). Por otra parte, nuestros resultados se encuentran en consonancia con estudios similares de Arabia Saudí, donde más del $90 \%$ de los residentes creían que debían participar en investigaciones, y que las mismas deberían formar parte obligatoria del programa de entrenamiento de su residencia médica (7).

Los médicos residentes paraguayos, participantes de este estudio piloto, presentan actitudes positivas y reconocen la importancia de la investigación, no obstante, desafortunadamente, sólo el $35 \%$ ha realizado investigaciones durante su programa de residencia. Lo anterior está en consonancia con investigaciones similares realizadas en Arabia Saudí7 y Pakistán (15), pero todavía lejos de lo evidenciado en países como Canadá, donde más del $50 \%$ de los residentes de Medicina Familiar participan activamente en proyectos de investigación durante su residencia1.

En la misma se observa que el mayor desafío para los médicos residentes de Medicina Familiar paraguayos es contar con un tiempo destinado a la investigación y, en segundo lugar, la falta de conocimientos y habilidades en investigación. Esto está en línea con lo evidenciado por otros investigadores en Canadá, que refirieron que en el $68 \%$ de los casos, el principal obstáculo para la investigación era la falta de tiempo de los médicos residentes (13).

En nuestro estudio, los tipos de estudios más comunes en los que los médicos residentes se involucraron fueron estudios descriptivos (30\%) y reportes de casos (15\%). Esto es similar a lo encontrado en otros estudios, como en Arabia Saudí, donde se encontró que los estudios descriptivos representaban el $32,8 \%$ de los trabajos en los que se encontraban involucrados los médicos residentes (7). Nuestros resultados reflejan que los médicos residentes no están lo suficientemente entrenados para realizar estudios distintos a los descriptivos o a los reportes de casos, como por ejemplo estudios de casos y controles o de cohortes, que requieren de mayor formación en metodología de la investigación.

Las características deseadas de los programas de residencia en Medicina Familiar que son efectivos en investigación incluyen, entre otros, tiempos protegidos para la investigación, programas de metodología de la investigación integrados al currículo, financiamiento, educación en metodología de la investigación y oportunidades para presentar los resultados de las investigaciones $(26,27)$. Es este sentido, a la luz de nuestros resultados, que consideramos que se deben dar pasos decididos a nivel del programa de residencia en Medicina Familiar de la Universidad Nacional de Asunción para involucrar a los médicos residentes en la investigación. Por ejemplo, el entrenamiento en metodología de la investigación debe ser una parte transversal y sistemática de los tres años que dura la residencia médica; asimismo, se debe asegurar que exista un tiempo protegido semanal para que los médicos se dediquen a la investigación. Por todo lo anterior, consideramos necesario el rediseño del currículo del programa de residencia en Medicina Familiar de la Universidad Nacional de Asunción.

Este estudio representa, hasta donde llega nuestro conocimiento, la primera investigación sobre las actitudes y los desafíos percibidos sobre la investigación de médicos residentes de Medicina Familiar de Paraguay, durante la residencia. Una limitación importante es que como este ha sido un estudio piloto, sus resultados pueden no reflejar la realidad de los 
médicos residentes de Medicina Familiar paraguayos en el campo de la investigación. Asimismo, el hecho de que nuestro estudio haya sido de corte transversal hace que los datos obtenidos representen una fotografía del momento y no una tendencia en el tiempo. Finalmente, como los datos fueron recolectados a través de un cuestionario autoadministrado, podrían no reflejar las tendencias verdaderas.

\section{REFERENCIAS BIBLIOGRÁFICAS}

1. Leahy N, Sheps J, Tracy S, Nie JX, Moineddin R, Upshur R. Family physicians' attitudes toward education in research skills during residency. Findings from a national mailed survey. Canadian Family Physician 2008; 54(3): 413-4. URL: http://www.cfp.ca/

2. Neale AV. A national survey of research requirements for family practice residents and faculty. Family Medicine 2002; 34(4): 262-7. URL: http://www.stfm.org/

3. Hebert RS, Levine RB, Smith CG, Wright SM. A systematic review of resident research curricula. Academic Medicine 2003; 78(1): 61-8. DOI: 10.1097/00001888-20030100000012

4. Alhaidera SA, Alshehric HA, Almedheshd SA. Research training, productivity and challenges among trainees of pediatric residency programs across Saudi Arabia. International Journal of Pediatrics and Adolescent Medicine 2015; 2(2): 70-4.

DOI: 10.1016/j.ijpam.2015.06.005

5. Abramson $M$. Improving resident education: what does resident research really have to offer? Transactions - American Academy of Ophthalmology and Otolaryngology. American Academy of Ophthalmology and Otolaryngology 1977; 84(6): 984-5.

6. Hillman BJ, Fajardo LL, Witzke DB, Cardenas $D$, Irion $M$, Fulginiti JV. Factors influencing radiologists to choose research careers. Investigative Radiology 1989; 24(11): 842-8. DOI: 10.1097/00004424-19891100000002
7. Mitwalli HA, Al Ghamdi KM, Moussa NA. Perceptions, attitudes, and practices towards research among resident physicians in training in Saudi Arabia. Eastern Mediterranean Health Journal 2014; 20(2): 99-104. URL: http://www.emro.who.int/emhjournal/eastern-mediterraneanhealth-journal/home.html

8. Smith M. Research in residency: do research curricula impact postresidency practice? Family Medicine 2005; 37(5): 322-7. URL: http://www.stfm.org/

9. Stockman 3rd JA. Research during residency training: good for all? The Journal of Pediatrics 2003; 143(5): 549-50. DOI: $10.1067 /$ S00223476(03)00450-5

10. Wood E, Kronick JB. A pediatric residency research curriculum. The Journal of Pediatrics 2008; 153(2): 153-4.

DOI:

10.1016/j.jpeds.2008.02.026

11. Souba WW, Tanabe KK, Gadd MA, Smith BL, Bushman MS. Attitudes and opinions toward surgical research. A survey of surgical residents and their chairpersons. Annals of Surgery 1996; 223(4): 377-83.

URL: http://journals.Iww.com/annalsofsurg ery/pages/default.aspx

12. Roth DE, Chan MK, Vohra S. Initial successes and challenges in the development of a pediatric resident research curriculum. The Journal of Pediatrics 2006; 149(2): 149-50. DOI: $10.1016 /$ j.jpeds.2006.05.001

13. Gill S, Levin A, Djurdjev O, Yoshida EM. Obstacles to residents' conducting research and predictors of publication. Academic Medicine 2001; 76(5): $477 . \quad$ URL: http://journals.Iww.com/academicme dicine/pages/default.aspx 
14. Cátedra de Medicina Familiar de la Universidad Nacional de Asunción. Programa y Planeamiento Educativo de la Residencia y Especialización en Medicina Familiar. Asunción: Editorial de la Facultad de CienciasMédicas; 2016.

15. Khan $\mathrm{H}$, Khan S, Iqbal A. Knowledge, attitudes and practices around health research: the perspective of physicians-in-training in Pakistan. BMC Medical Education 2009; 9: 46. DOI: $10.1186 / 1472-6920-9-46$.

16. Dirección Académica de la Universidad Nacional de Asunción. Malla curricular de la Carrera de Medicina y Cirugía. Asunción: Editorial de la Facultad de Ciencias Médicas; 2016.

17. Ullrich N, Botelho C, Hibberd $P$, Bernstein $H$. Research during pediatric residency: predictors and resident-determined influences. Academic Medicine 2003; 78: 12538. DOI: $10.1097 / 00001888-$ 200312000-00014

18. Logan PM. Resident research in Canadian radiology training programs. Annals of the Royal College of Physicians and Surgeons of Canada 1998; 31: 233-5.

19. McCrindle BW, Grimes RB. Will pediatric residents do research? A survey of residents' attitudes. Annals of the Royal College of Physicians and Surgeons of Canada 1993; 26: 283-87.

20. Buschbacher R, Braddom RL. Resident versus program director perceptions about PM\&R research training. American Journal of Physical Medicine and Rehabilitation 1995; 74: 90-100. URL: http://journals.Iww.com/ajpmr/pages /default.aspx
21. Temte JL, Hunter PH, Beasley JW. Factors associated with research interest and activity during family practice residency. Family Medicine 1994; 26: 93-7. URL: http://www.stfm.org/

22. Morris BA, Kerbel D, Luu-Trong N. Family practice residents' attitudes toward their academic projects. Family Medicine, 1994; 26: 579-82. URL: http://www.stfm.org/

23. Fitz-Gerald $M$, Kablinger A, Manno B, Carter O, Caldito G, Smith S. Psychiatry residents' participation in research: a survey of attitudes and experience. Academic Psychiatry; 2001, 25(1): 42-7. URL: http://link.springer.com/article/10.11 76/appi.ap.25.1.42

24. Gay SB, Hillman BJ. Evaluation of a mandatory radiology resident research rotation. Academic Radiology 2000; 7(3): 172-5. DOI: 10.1016/S1076-6332(00)80119-1

25. Silcox L, Ashbury T, VanDenKerkhof E, Milne B. Residents' and program directors' attitudes toward research during anesthesiology training: a Canadian perspective. Anesthesia and Analgesia 2006; 102(3): 859-64. DOI:

10.1213/01.ane. 0000194874.28870 . $\mathrm{fd}$

26. Mills OF, Zyzanski SJ, Flocke S. Factors associated with research productivity in family practice residencies. Family Medicine 1995; 27(3): 188-93. URL: http://www.cfp.ca/

27. DeHaven MJ, Wilson GR, O'ConnorKettlestrings $P$. Creating a research culture: what we can learn from residencies that are successful in research. Family Medicine 1998; 30(7): 501-7. URL: http://www.cfp.ca/ 